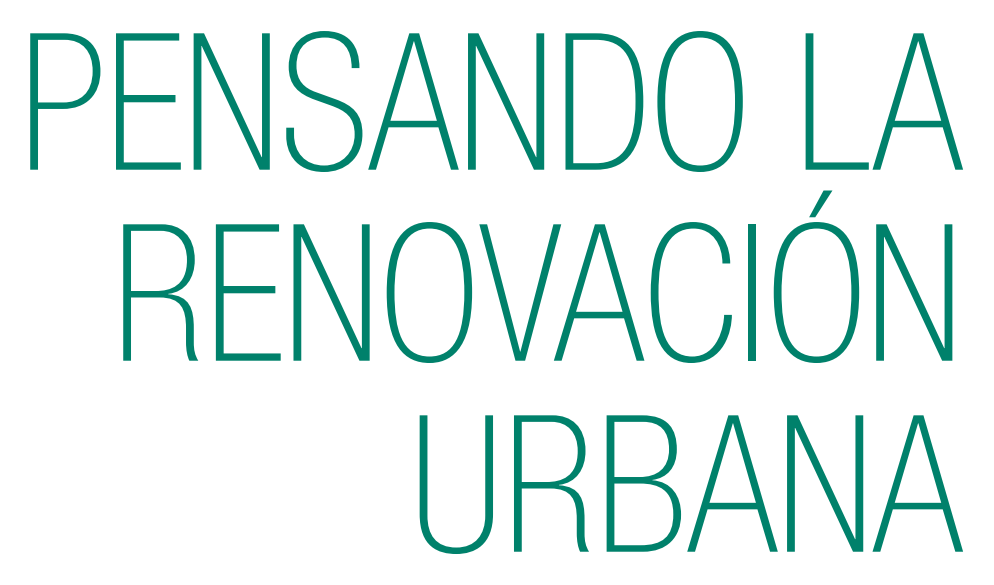

\title{
en el contexto de la
}

ciudad latinoamericana

\section{Carlos M. Yory-García \\ Universidad Católica de Colombia}

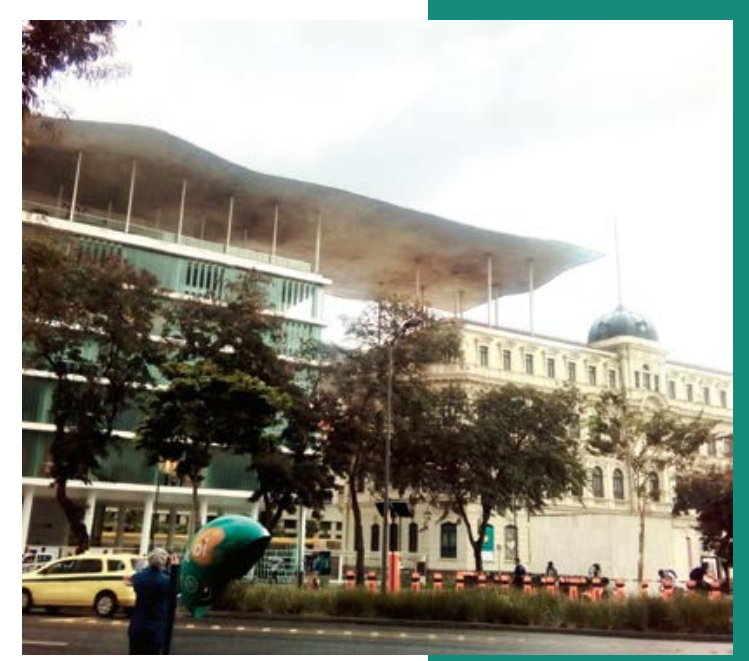

Panorámica centro Rio de Janeiro, 2018. Carlos M. Yory-García. 
El primero fue el Sol del Agua y pereció ahogado

El segundo se llamó Sol de Tierra, y lo devoró, como una

bestia feroz, una larga noche sin luz

El tercero se llamó Sol de fuego, y fue destruido por una

lluvia de llamas

El cuarto fue el sol de Viento y se lo llevó un huracán

El quinto sol es el nuestro, bajo el vivimos, pero también el desaparecerá un día, devorado, como por el agua, como por la tierra, como por el fuego, como por el viento, por otro temible elemento: el movimiento.

El quinto sol, el sol final, contenía esta terrible advertencia: el movimiento nos matará... Carlos Fuentes Macías

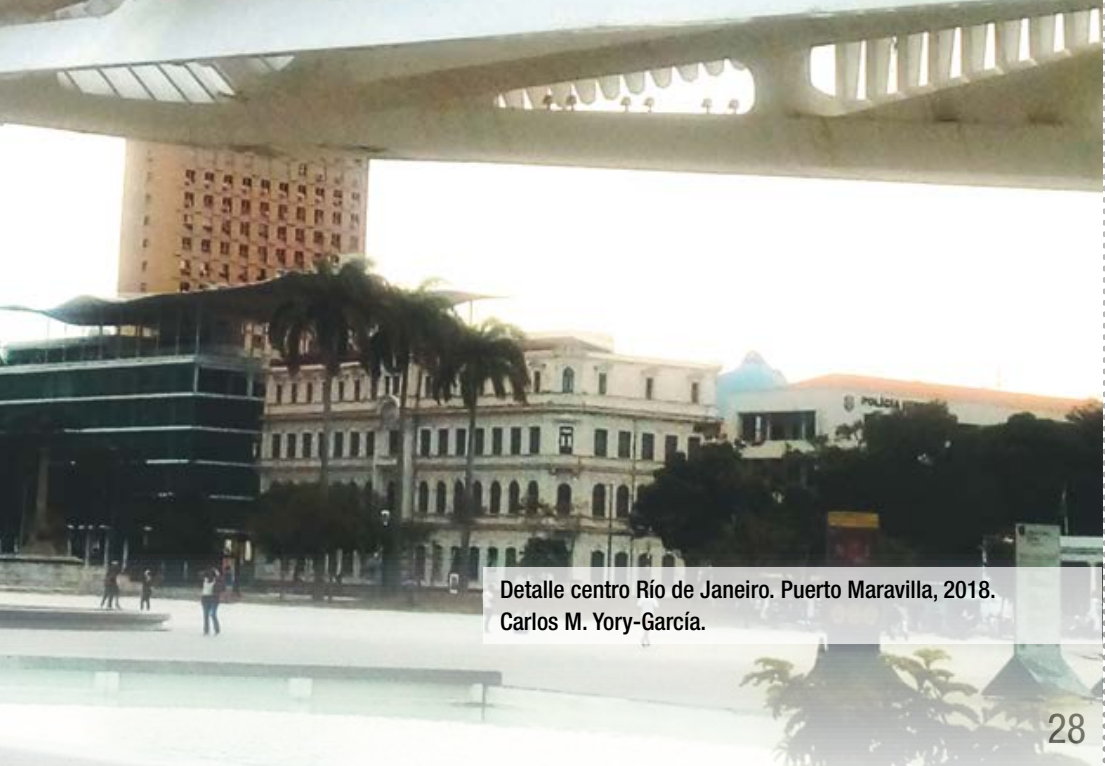

En su libro Los cinco soles de México, Carlos Fuentes analiza la relación entre creación y destrucción que, en su opinión, caracteriza desde siempre la historia de México y, en nuestra opinión no solo la de América Latina, sino más exactamente la de la ciudad latinoamericana; particularmente en lo que tiene que ver con la vivencia de un pasado común enraizado tanto en el mito, en el rito y en la fiesta como en el colonialismo, en el cristianismo, en el asistencialismo y en la dependencia.

Los aztecas pretendieron explicar el conflicto y su juego de fuerzas, es decir, "el tiempo humano" a través de una sucesión de cinco soles que a partir de la indisoluble relación que planteaban entre creación y destrucción, de esta forma se iban sucediendo.

Así, en el marco del azaroso contexto de incertidumbre que acompaña nuestra época, hecha de relaciones y de situaciones efímeras donde, no hay duda, las cosas se hacen para no durar, no podemos menos que afirmar que, en medio de nuestras raíces, el quinto sol, más que haber llegado para instaurar el cambio, en realidad nunca se ha ido. Un cambio que, no obstante, no es el de una cosa hacia otra, sino que es el de un estado de cosas en sí mismas cambiantes, tal como corresponde entender la instauración de un orden que, como contexto, piso y principio de realidad, resulta ser el cambio mismo.

Hablamos pues de una amenazante idea de cambio con la cual siempre hemos vivido. Idea que, dependiendo de nuestra manera de enfrentar el movimiento que trae consigo, podrá o no, arrancarnos del piso sacándonos del tiempo atropellados por la prisa, o excluyéndonos del espacio común 
compartido; en el primer caso, borrándonos de la memoria, en el segundo, arrojándonos del lugar que hemos construido.

Acaso el patrimonio que localmente nos caracteriza no sea otra cosa más que un manojo de hojas que tercamente permanecen aferradas a un árbol, que si bien puede tener profundas raíces, cada vez se encuentra más azotado por el viento de la globalidad ¿Aire de cambio u oportunidad para continuar creciendo? ¿Viento desgarrador que todo lo arrasa o renovador aire fresco? ¿Fin de los tiempos o nueva oportunidad para lograr existir insistiendo? Acaso llegó el momento de decir, como Shakespeare en Romeo y Julieta, "el amanecer es tan tarde que podemos decir que es temprano", todo depende de cómo nos ubiquemos en ese umbral del tiempo: aferrándonos a la noche, por ser lo que conocemos, o dándole la bienvenida a las sorpresas que nos traerá el nuevo día.

El asunto es ¿Nos encontramos al final de una época que no termina o al comienzo de otra que difícilmente comienza? ¿La oscuridad termina o apenas está comenzando disfrazada de nuevo día? Como quiera que sea, tenemos tantos retos que enfrentar que no nos queda más que echar a andar para superarlos.

No obstante, y en medio del juego de fuerzas que suponen actualmente las interacciones entre lo local y lo global, ¿Hasta dónde nuestro pasado nos determina y, si es así, de qué manera lo hace? ¿Cuándo "termina" nuestro pasado para poder afirmar que, a partir de allí, podemos proyectar nuestro futuro? ¿Hasta dónde aquello que nos caracteriza puede llegar a constituir una "ventaja comparativa" al interior de un mundo cada vez más indiferenciado?

¿Y si, finalmente, no estamos determinados por nuestro pasado sino constituidos por él en medio del cambio? Si esto es así, el cambio no es el enemigo sino la posibilidad misma de afianzarnos en lo que somos una vez hayamos dejado de lado aquello que hemos dejado de ser; esto a condición de que superemos el imperialismo del determinismo histórico que nos ata a un pasado inmutable. A fin de cuentas, no somos lo que heredamos, sino lo que hacemos con lo heredado; de ahí que nuestro patrimonio esté menos en las cosas que en la relación que establecemos con ellas.

De esta forma, entender lo que somos supondría la necesidad de examinarnos en medio de los acontecimientos y a la luz de nuestro papel dentro de ellos, lo cual nos lleva a entender la realidad como un constructo y no como un inexorable factum brutum, como un hecho ineludible y pre-determinado impuesto desde fuera.

En este sentido y por el hecho de que, al decir de Octavio Paz, América Latina encuentra su futuro en el pasado, no contempla la idea de un final o, menos aún, la de un sentido. Por lo mismo, tampoco tiene un inicio sino que posee un origen que, he ahí su particularidad, no se haya en el tiempo sino más allá de él, in illo tempore, en el ámbito del mito, en aquel lugar desde donde, en el imaginario popular, todas las cosas proceden, perseveran y se mantienen en medio del cambio trascendiendo, incluso, la historicidad. 
No obstante, la base de ese origen preyace en un inestable y conflictivo fondo telúrico, allí se asienta nuestra ambigua naturaleza atada tanto a la certeza del cambio como a la incertidumbre que provoca la movilidad. No es gratuito que así como su geografía "natural" está marcada por contrastes (desiertos, selvas, montañas, llanuras y valles), su geografía "histórica" y, con ella, su geografía urbana, lo esté por fragmentos, por desgarramientos, por discontinuidades y por rupturas.

De esta forma, la manera latinoamericana de transformar el paisaje natural da cuenta de lo más natural de la condición latinoamericana: el movimiento, el contraste y el accidente que en consecuencia caracteriza nuestro hábitat común compartido. Un movimiento que, no obstante, tiene tanto un modo como un precio; el modo: el conflicto, el precio: el cambio.

Latinoamérica es el conflicto en estado puro; un conflicto que, a pesar de darse, como todos, sobre y por el territorio, en procesos permanentes de territorialización, desterritorialización y reterritorialización (Haesbaert, 2004), tiene una particularidad $\mathrm{y}$ es que este no espera ser resuelto sino apenas conciliado: Dionisos frente al crucificado, Apolo frente a Dionisios, Queztalcoatl frente a Huitzilopotzli; el trámite y la gestión del conflicto es el gran tema latinoamericano.

Desde esta perspectiva, la naturaleza telúrica de América Latina, y con ella la geografía de la ciudad latinoamericana, no está determinada por su pasado sino constituida por sus accidentes, en su manera de darse como ocurrencias en medio del conflicto, de ahí que lo suyo sea el acontecimiento que causa el accidente y no la pasiva adaptación a un accidente predeterminado. Al fin y al cabo, el acontecimiento que marcan el mito, el rito y la fiesta constituye el espacio de la emergencia en la cual habita lo latinoamericano. Emergencia en un doble sentido: porque aparece dando lugar a un nuevo orden y porque ese nuevo orden comporta un cambio que trae consigo unas inesperadas y no necesariamente felices consecuencias.

De esta forma, lo emergente constituye un espacio en sí mismo que no puede identificarse a través de la relación entre un pasado y un futuro, sino mediante el conflicto entre un antes y un después en el que surge el tiempo intenso, el tiempo inmenso y el tiempo profundo que, desde la cosmogonía Aymara, marca nuestra vida a cada paso, como nos recuerda la fiesta mexicana de los muertos que de tal forma trasciende la idea de difunto reemplazándola mágicamente por la de ante-pasado; la muerte no existe, parece decir lo latinoamericano. Ahora bien, si la muerte no existe ¿Qué esperar de lo urbano que se resiste al cambio en medio de la movilidad?

En este sentido, el origen condiciona lo latinoamericano en su manera de obrar pero no determina sus resultados, por esto, ni el determinismo histórico ni el determinismo geográfico logran dar cuenta de lo latinoamericano. Es nuestra manera de obrar la que da forma a nuestro paisaje y no al revés, el verdadero patrimonio está en la gente; no obstante, esa "manera de obrar" está condicionada, no solo por un "origen" (que nos pone a mirar y a sentir 
de una determinada manera), sino por el propio juego de fuerzas en el que inexorablemente nos encontramos inmersos: el mundo global, las homogenizadoras pautas de consumo, la hibridación cultural, el debilitamiento del Estado-nación y las implacables leyes de mercado.

Desde aquí, la globalización no puede ser un imperativo que determine y condicione lo latinoamericano a través de unos usos, unas prácticas y unos lenguajes, sino tan solo un marco con el que necesariamente se debe interactuar.

Hace ya varios años Octavio Paz anticipaba en el Laberinto de la soledad (1981) de los análogos laberintos en que la Ilustración metía a la razón imponiéndole a la Historia — como teleológicamente quería Hegel- tanto un sentido (común para todos) como una orientación; la cual, consideramos, debería entenderse desde una triple connotación, en cualquier caso mítica, que es necesario superar: "la del progreso basado en el alejamiento de lo que, frente a lo nuevo (moderno), va quedando atrás; la de la continuidad lineal de los acontecimientos y felizmente causal de un futuro cierto (aquel al cual nos dirigimos); y la de la acumulación gracias a la cual no somos, sino que devenimos seres históricos" (Yory, 2009, p.5) en este último caso orientados en nuestro presente a "ser más" en relación directa no tanto con lo que podamos poseer, sino con la manera como hacemos visible eso nuevo que poseemos o que creemos poseer.

Después de todo, mejor que tener es aparentar, puesto que la apariencia nos libra de toda respon- sabilidad descargándonos de un peso histórico. Las decisiones: es mejor que otros las tomen, que sean ellos los que sean juzgados.

Desde aquí, para la latinoamérica del Nobel mexicano, "el pasado, lejos de recordarse como un patrimonio muerto que debe inmortalizarse de una $u$ otra manera (papel del monumento, o mejor, de la monu-mentalidad), pervive a través de una memoria que no se cansa de traerlo a la fiesta, lo que equivale a decir: de traerlo a la calle" (Yory, 2009, p.5). He ahí una gran diferencia entre esa denominación de "patrimonio" que, para ser recordado, en tanto con-memoración, debe dejarse monumentalizar a través de las cosas - por naturaleza, evidentemente muertas- y el patrimonio que de tal suerte puede denominarse "vivo", en tanto su razón de ser no estriba en su materilialidad sino en la relación que guardamos con esta.

Al fin y al cabo, como evidencia el referente mexicano, ¿No existe acaso un innegable sincronismo entre los múltiples aspectos, dimensiones y ejemplos de su patrimonio que hacen de este un $\mathrm{co}^{-}$ herente - aunque sincrético y mestizo- paisaje cultural que de tal forma acusa una clara identidad? Si esto es así, ¿Qué es entonces, lo que en atención a las fuerzas del mercado, disfrazadas de contemporaneidad, debemos renovar?

Si asumimos que la ciudad es una agrupación de edificicaciones, infraestructuras, servicios y equipamientos, y que lo urbano es una idea, un significado más o menos común que da cuerpo a un conjunto de identidades, imaginarios, formas de relación, prácticas culturales y significados relacionados con unas 
formas definidas de organización en lo político, en lo social, en lo ambiental, en lo económico y en lo cultural, tendríamos que aceptar que la ciudad y lo urbano son dos cosas distintas, donde la primera no solo no supone de facto, lo urbano, sino que tampoco comporta la urbanidad, entendida como un forma responsable de interacción entre los seres humanos, la naturaleza, el Estado, el mercado, el entorno construido y la bistoria en cuanto tal.

¿A qué nos referimos, entonces, cuando hablamos de renovación urbana? ¿Por qué confundimos la renovación de la imagen de la ciudad con la renovación de lo urbano mismo? ¿Será que acaso no hay error y que lo que la globalización pretende con las ciudades a través de las estrategias de renovación que acompañan los procesos de city marketing es apuntarle a la renovación de lo urbano en cuanto tal? ¿A la transformación de aquello que en el escenario de las ciudades - aunque no de forma exclusiva allí- nos constituye de la manera más propia como una peligrosa diferencia que, por lo mismo, atenta contra el homogenizante principio de razón que impone el nuevo orden mundial?

Se nos ha querido inculcar que las ciudades son peligrosas cuando en verdad lo que es temible para cualquier sistema es lo urbano mismo, aquello que difícilmente se deja gobernar. No nos engañemos, la transformación de las ciudades que acompaña los procesos de renovación urbana le apuesta a la transformación y control de la urbanidad. Al fin y al cabo ¿Qué otra cosa es la planeación sino un instrumento de tecnología política al servicio del principio de razón dominante?

Por lo anterior, se debe diferenciar en los procesos relacionados con la renovación urbana "entre la gestión urbana propiamente dicha y la gestión de la ciudad en atención a la propia divergencia que existe entre renovar la imagen física, compromiso de la gestión de la ciudad, y renovar su dinámica estructural, responsabilidad primera de la gestión urbana propiamente dicha" (Yory, 2009, p.7).

De esta suerte, hacer renovación urbana resulta ser algo muy diferente a alentar la estricta "renovación de la ciudad", dado que si bien la primera involucraría un compromiso concertado de renovación social y humana derivado de la intervención directa sobre la estructura portante de la ciudad, la segunda se conforma tan solo con llevar a cabo una intervención - muchas veces cosmética- que a lo más apunta a presentar una "apariencia renovada de esta" impasible, normalmente, frente a la deuda social que tal imagen suele generar.

A fin de cuentas, de lo que se trata es de atraer el capital y para ello basta con colocar los adecuados anzuelos que hablen y promocionen una imagen de prosperidad material en el marco de lo que en otros trabajos denominamos la fashion city o la ciudad-postal.

Una ciudad que de tal suerte vende y se vende gracias a la ayuda de una enlucida envoltura cargada de modernismo pero en muchos casos carente en todo de modernidad. Para el efecto basta con adoptar los códigos escenográficos y asépticos de esta: edificios 
en altura, espacios públicos relucientes, tintes de verde, infraestructuras deslumbrantes, arte urbano, concentración de servicios y, entre otras cosas, iluminación refulgente. Imagen de bienestar material que frecuentemente deja de lado las dinámicas sociales y culturales, los sistemas tradicionales de intercambio e interacción, las expresiones espontáneas de vida y, muchas veces, los lugares de identificación, recurrencia y significación aceptados y construidos a lo largo del tiempo por la ciudadanía, lo cual supone que:

El resultado de esta manera de entender la "renovación”, en la que rápidamente están cayendo buen parte de nuestras ciudades, es una imagen renovada de éstas que, no solo termina por homogenizar el paisaje urbano borrando muchas veces las naturales diferencias entre una y otra ciudad, sino que frecuentemente poco o nada tiene que ver con las dinámicas históricas tradicionales e incluso patrimoniales que marcan sus diferencias y sus acentos, las que, no obstante, por el solo hecho de existir no necesariamente habrían de ser conservadas pues muchas de ellas atentan contra el uso democrático, las nuevas demandas sociales o incluso la seguridad, pero si, al menos, habrían de ser tenidas en cuenta y valoradas desde la perspectiva de sus usuarios y habitantes en función de lo que éstos desean. Después de todo, cómo preguntáramos en un trabajo anterior ¿Qué clase de renovación urbana sería aquella que dejase de lado los problemas estructurales de la ciudad, sus tejidos sociales y la opinión de sus ciudadanos? (Yory, 2009, p.9).

En este sentido, la gestión social e integral del territorio tendría que cumplir un importante papel a la hora de promover y concretar, al interior de consecuentes operaciones urbanas, procesos concertados de renovación que en primer lugar definan con la gente qué es lo que se debe renovar a la luz de la identificación de un "paquete" de afirmaciones y transformaciones definidas por ella, para que en cualquier caso la renovación responda a:

\footnotetext{
Un espíritu tan correctivo como preventivo. En el primer caso, interviniendo directamente sobre las causas del deterioro y no solo sobre sus signos ciertos. En el segundo, apoyándose en el acervo patrimonial, tanto físico como social, con el que cuenta la ciudad. Lo que se buscaría, finalmente, sería generar dinámicas pedagógicas capaces de coadyuvar en la construcción de un espíritu colectivo de corresponsabilidad social e individual que haga sentir a sus habitantes que la ciudad les pertenece (Yory, 2009, p.9).
}

Desde aquí, uno de los retos más importantes para la renovación urbana, a partir de un enfoque patrimonial, no necesariamente comprometido con el conservacionismo, es el de promover la adopción de enfoques integrales que en cualquier caso respondan, tanto a la dimensión físico-espacial de la misma, como a su correlato cultural, ambiental, económico, social y político, esto con el fin de prevenir y/o enfrentar la expulsión de población (gentrificación) y el desplazamiento.

De esta forma, los procesos de renovación urbana tendrían que acompañarse de dinámicas concretas y comprometidas de renovación de lo urbano en cuanto tal derivadas de una clara articulación entre modelo de ciudad y modelo de gobierno donde el ciudadano sea el punto medio que otorgue a dichos procesos tanto realidad como sentido; es decir: "Una renovación que no 
solo se encargue de enfrentar el deterioro físico y social de la ciudad, sino de prevenirlo al propiciar nuevas dinámicas tanto sociales como económicas en el marco de una renovada relación con "el otro", con la institucionalidad, con la naturaleza, con el mercado, con la historia y con el mundo en general" (Yory, 2009, pp.7-8).

Al fin y al cabo, la ciudad es una geografía simbólica y por tanto construida en el tiempo gracias a todo un sistema de interacciones (Santos, 1996). Un espacio común colectivo hecho de encuentros y desencuentros, de acciones y reacciones, de apuestas y de resultados que si bien preteden ser previsibles, las más de las veces resultan aleatorios e impredecibles.

En este contexto - particularmente en el caso de la ciudad latinoamericana- surge una particular relación entre conservación del patrimonio y renovación urbana. No se trata de hacer una cosa u otra, sino de poner a operar ambas a la luz de un único objetivo: dar rienda suelta al gran negocio del capitalismo que consiste en la destrucción de espacio gracias a las oportunidades de generación de suelo útil que esto genera, bandera que acompaña la demolición de áreas enteras de la ciudad para alentar la movilidad del capital.

La tarea de la renovación urbana es clara: generar atractivo para la inversión por la vía de la generación de espacio — valga decir de suelo- en el cual se haga evidente la movilidad de los usos y, tanto mejor, el tráfico político de la significación. La que, mal que bien, igual que la renovación, atrae al turismo - punto de encuentro entre renovación y patrimonio - al jugar con una idea funcional y acomodaticia de este útimo, frecuentemene reciclado y, en muchos casos, disneylandizado y puesto al servicio de la renovación.

De ahí que sea indispensable crear el espacio donde no existe o, lo que es lo mismo, removerlo a tal punto que, al ser re-novado, se haga irreconocible o, mejor, resulte tanto más parecido a esos otros espacios que a nivel global, por cosmopolitas, han probado ser atractivos al uso - valga decir al consumo- y a la inversión, cuanto menos recuerden el pasado provinciano del cual provienen y han surgido. Lo cual exige apelar a la renovación, aunque para ello haya que echar abajo sectores enteros de ciudad. En el marco de una economía de casino, la desaparición de la oferta aumenta la demanda.

Aquí, el verdadero patrimonio resulta ser el suelo urbano, oportunista o parasitariamente beneficiado por las características de un entorno atractivo, lo cual ocasiona que el valor de uso del espacio - en gran medida detentor de ese atractivo- devenga en valor de cambio y rentabilidad. De esta inversión axiológica donde el valor de cambio reemplaza al valor de uso se deriva que sea el precio quien ahora dote de valor al espacio. Un espacio de tal suerte re-novable y, por tanto, "entrado en valor" gracias a su nuevo estatuto como atractor preferente y protagónico para la inversión.

\footnotetext{
Así, se invierte para atraer un plus valor, el que proporciona una cadena de nuevas inversiones, aquellas desde las cuales las llamadas operaciones urbanas devienen en simples desarrollos
} 
inmobiliarios cuyo manejo de plusvalías, así como de cargas y beneficios, se suele quedar, en gran medida, en el sector privado pues es éste quien ha facilitado la inversión. Desde aquí, se renueva para conservar, en este caso no un bien patrimonial, sino un orden de cosas inmerso en la lógica del capital (Yory, 2009, p.6).

Dentro de esta lógica, en cualquier caso, hegemónica, también se conserva para renovar, dado que la conservación solo tiene lugar si ésta supone una escenografización rentabilizable del espacio por la vía de su reutilización, la que de tal suerte entra a capitalizar una imagen proclive, en consecuencia, a la renovación.

Solo así la renovación aceptará adecuarse al "espíritu del lugar" en tanto este no vaya acompañado de la conservación de su población original, la que tendrá que ser desplazada en tanto la misma detente algún signo de pobreza, "afeamiento", marginalidad o incapacidad de adaptación al espíritu de los tiempos que impone la era del consumo, la inversión y la rentabilidad.

Al fin y al cabo, hacer renovación en áreas de conservación comporta un doble propósito que en consecuencia hace doblemente atractiva la inversión: por un lado, servirse del contexto patrimonial para obtener beneficios tributarios, prediales y en el pago de servicios; por otro, enmarcar la inversión en un contexto, no solo de alta valorización, sino harto atractivo para el turismo y, por lo mismo, para el uso y consumo de una población desarraigada e itinerante, la cual solo exige que el entorno le ofrezca las condiciones de seguridad, movilidad, calidad ambiental, equipamientos y servicios que, amén de su paisaje urbano, consolide e incremen- te su atractivo. Un atractivo que, a ojos del turista, lejos de suponer el desplazamiento de la población tradicional — como parecen entender muchos de los renovadores urbanos- se encuentra íntimamente unido a ella por cuanto, desde el ejercicio de sus prácticas culturales, constituye parte integral de un indefectible todo.

$\mathrm{El}$ asunto es que, asumiendo que la gentrificación como se denomina este proceso de desplazamiento de población derivado de los procesos de renovación urbana - no sea un objetivo directo de la renovación, sino una lamentable consecuencia, comparable a los efectos colaterales de los bombardeos estadounidenses en Irak que no iban dirigidos a la población civil pero que en muchos casos arrasaban con ella, el hecho es que, no solamente el desplazamiento continua ocurriendo, sino que pareciera hacer parte de la política pública toda vez que, salvo escasas ocasiones, la manera como se concibe la misma - de la mano del tono de las operaciones urbanas que a ella responden- no suele ir acompañada de claras estrategias capaces de amortiguar, en el bolsillo de los residentes, el incremento en el costo del suelo que supone el aumento de la valorización.

De otra parte la globalización, valiéndose en gran medida del turismo urbano, pero, también, de las inescrutables leyes del mercado, hace que en vez de proponer, por ejemplo, circuitos que integren e interconecten los distintos entornos urbanos patrimoniales, hace que estos compitan poniéndolos de tal suerte a "facturar" individualmente dentro de 
los consabidos procesos de city marketing desde los cuales se promocionan hoy en día las ciudades.

En este contexto, la "marca-lugar" es lo que cuenta y el patrimonio, como el vino, adopta una particular "denominación de origen" desde la cual toma lugar en las consecuentes redes que de tal suerte mueven la economía global, motivo por el cual "el patrimonio se convierte en una de las principales ventajas comparativas de las ciudades en el concierto internacional" (Yory, 2009, p.6) de lo que en consecuencia sería su "puesta en venta" gracias a la exotizacion comercializable de sus referentes particulares y sus diferencias.

Sin embargo, si por un lado la modernidad triunfante que impone la globalización cree fielmente en el paradigma de la innovación, el cambio y, por lo mismo, de la re-novación —asi sea de lo mismo-, por otro, la ciudad latinoamericana -oscilante siempre entre la pre-modernidad y la posmodernidad dado que nunca se ha sentido cómoda del todo con la modernidad - se resiste, menos al cambio que a la movilidad que este exige, pues a pesar de sus signos modernizantes se encuentra anclada, desde illo tempore, en un juego de valores, lógicas y razones en todo distintas a las que sin más promueve la que a sus ojos resulta ser la lógica extraña de la modernidad (Yory, 2012).

Así, latinoamérica continúa apostándole al carnaval, al festejo, al evento, al ritual y al encuentro más que al rendimiento óptimo y a la funcionalidad. Papel que, más allá del desarrollo de actividades espontáneas o no que en diferentes espacios recuerden y renueven este insobornable fondo telúrico y, por lo mismo, eventual, que alienta y anima al latinoamericano, encuentra en la calle-lugar, y no en la calle-distancia, su razón de ser y su mejor vehículo de transmisión, cultivo y desarrollo. La calle es, sin lugar a dudas, el lugar dilecto de la informalidad, pero no solo de aquella que alude a la economía de subsistencia, sino sobre todo de aquella otra que tiene que ver con una particular manera de ser y de estar viviendo al margen y, por tanto, más allá de cualquier forma contingente de formalidad.

Aquí radica el valor de la calle y, en consecuencia, el sustantivo valor del espacio público como detentor del propio derecho a la ciudad (Lefebvre, 1969) ¿Cómo concebir desde aquí una idea de renovación urbana fundamentada en la pérdida de los referentes? Al fin y al cabo, frente a los procesos de desterritorialización y de homogenización del paisaje de la ciudad que la renovación suele traer consigo, se reterritorializa fragmentando el espacio y por lo mismo marcándolo socialmente (Santos, De Souze y Silveira, 1994).

\footnotetext{
Gabriel García Márquez (...) señalaba en un pasaje de Cien años de soledad como, ante la llegada recurrente, periódica e inevitable de la epidemia del olvido a las calles y casas de Macondo, sus habitantes reaccionaban, previamente, marcando todas las cosas para no olvidarse de su nombre, solo que olvidaban que al llegar la epidemia del olvido también se les olvidaría leer.

¿No traerá al mundo la globalización, y su espíritu homogenizador, los aires de la epidemia que, año tras año, llegaba a las calles de Macondo? ¿En medio de ella, cómo habremos de nombrar las cosas que antes nos representaban? ¿A través de que signos $\mathrm{y}$, sobre todo, mediante que lenguaje identificaremos aquello
} 
que, en medio de la indiferenciación, nos nombra? ¿Cómo vamos a poder leer si aceptamos perder nuestro lenguaje? La pérdida de la referencia no puede significar menos que la propia pérdida de la identidad (La cursiva es nuestra. Yory, 2009, p. 7).

Si bien la globalización ha demostrado que se desterritorializa homogeniezando, es decir, colonizando el espacio, lo que latioamérica demuestra por todas partes es que se reterritorializa fragmentando, esto es, apropiándose del territorio, rayándolo y haciéndolo suyo (Prévôt, 2000).

Sea este el valor patrimonial de los zapatos viejos (Heidegger, 1993): adecuarse a los pies después de que estos, de tanto andar, los han hecho suyos, no son nuestros por haberlos comprado sino por haberlos andado. El zapato viejo es un monumento vivo que de tal forma narra y da cuenta de una vida recorrida, de una vida hecha paso a paso. Mientras que el zapato nuevo talla en tanto nos adaptamos a él, el viejo se adecua al pie como si se tratara de una continuidad de nuestro cuerpo. No es claro si nos adaptamos a él o él a nosotros, ya que si bien nos acomodamos a este, el paso del tiempo - a través del cual damos cuenta de nuestro propio paso-, nos marca tanto como el zapato que de tal suerte testimonia nuestro andar y, por lo mismo, nuestra forma de vida. No solo le damos forma a través de nuestros pasos, sino que los mismos quedan registrados a través de aquel, al punto que tales pasos terminan siendo condicionados, muchas veces, por este. Renovar nuestros zapatos supone renovar nuestro andar, lo cual no tendría que significar seguir otros pasos o, peor aún, tomar otro camino.
Haesbaert, R. (2004). O mito da desterritorialização. Rio de Janeiro: Ed. Betrand. Brasil.

Heidegger, M. (1993). Construir, habitar, pensar. En (s.ed.) Ciencia y Técnica (3a ed.) (pp. 197-223). Santiago de Chile: Ed. Universidad Santiago de Chile.

Lefebvre, H. (1969). El derecho a la ciudad. Barcelona: Ed. Península.

Paz, O. (1981). El laberinto de la soledad. México: F.C.E.

Prévôt, M.F. (s.m, 2000). América Latina: la ciudad fragmentada. Revista de Occidente. (232), 25-46.

Santos, M. (1996). Metamorfosis del espacio habitado. Barcelona: Ed. Oikos-Tau.

Santos, M., De Souze, M. A., y Silveira, M. L. (1994). Territorio, Globalizaçâo e Fragmentaçâo. São Paulo:Ed. Hucitec.

Yory, C.M. (2012). Pensamiento crítico, globalización y patrimonio: una aproximación desde la noción de paisaje cultural. En Niglio Olimpia (Ed), Paisaje cultural urbano e identidad territorial (pp. 5-22). Roma: Aracne Editrice.

Yory, C.M. (diciembre, 2009). Del monumento a la ciudad: una mirada a la valoración patrimonial más allá de la monu-mentalidad. Revista EDA. Esempi di Archittetura. Recuperado de www.esempidiarchitettura.it/.../oggetti_articolo_127_ITA_JNV3fSSaYE2eXc59J5x4B. 


\section{CONTENIDO}

Introducción .................................................................... 40

Parte 1. Historia crítica de la renovación urbana .................4 43

Fase 1. Higienismo .................................................4 43

Fase 2. Conservacionismo ......................................... 47

Anti-conservacionismo ............................................. 49

Fase 3. Ciudadanía .................................................... 50

Movimientos vecinales: habitabilidad. ........................51

Movimientos contraculturales: calidad de vida ...............52

Fase 4. Renovación integral ..........................................55

Parte 2. Situación actual y perspectivas de

la renovación urbana ..................................................... 58

a. Nuevas funciones de lo urbano ....................................59

b. Nuevos requerimientos y/o desafíos ............................62 62

c. Nuevas condiciones de gestión..................................66

Referencias...................................................... 71

Goycoolea, R. (2019). Renovación urbana. De la certeza a las incertidumbres. En Yory, C. M. (Ed.), Renovación urbana. Globalización y patrimonio (pp. 39-73). doi: 10.14718/9789585456624.2019.3

1. Las reflexiones aquí presentadas se apoyan, sobre todo, en el trabajo realizado en el seminario Criterios contemporáneos de renovación urbana, que el autor dirige desde hace una década en el Master Universitario de Proyectos Avanzados de Arquitectura y Ciudad, MUPAAC, Universidad de Alcalá (España)

2. Arquitecto por la U. Técnica del Estado (Chile, 1983); Dr. Arquitecto, U. Politécnica, Madrid (1992). Experiencia académica: Coordinador del Doctorado Internacional en Arquitectura y Urbanismo (México-España, UPM/U. Veracruzana, 1994-98). Fundador (1999) de Escuela de Arquitectura de la U. de Alcalá y su primer Director. Hoy es Profesor Titular de Análisis de Formas Arquitectónicas en ella. Tiene publicaciones sobre sus proyectos e investigaciones, centradas en la concepción y percepción del espacio habitable, en libros y/o revistas de 11 países. Miembro Comité Científico de la RIGPAC. Correo: roberto.goycoolea@uah.es. 